\title{
Operando TEM - Detection of Gas Phase Catalysis in an Environmental TEM with EELS
}

\author{
S. Chenna, ${ }^{*}$ and P.A. Crozier \\ * School of Mechanical, Aerospace, Chemical and Materials Engineering, Arizona State University, \\ Tempe, AZ, 85287-6106
}

In-situ environmental transmission electron microscopy (ETEM) is a powerful tool for obtaining atomic scale structural and chemical information under reacting gas cond itions. We have shown the significance of in-situ ETEM studies in developing the struct ure-activity relationship in supported metal catalysts for heterogeneous catalysis [1]. However in this case, the catalytic measurements were performed in an ex-situ reactor. Ideally we would like to perform an operando experiment where we o bserve the nanostructural chang es taking place in the c atalyst while s imultaneously measuring the catalyst activity and selectivity. We have shown that the reactant gas com position around the TEM sam ple can be m onitored by using electron energy-loss spectroscopy (EELS) [2]. Here we demonstrate that EELS can also detect product gas for mation over a catalyst thus opening up the possibility of perfor ming true operando microscopy. As a preliminary test we perform ed CO oxidation on a Pt m esh inside the ETEM, we w ill extend this approach for studying catalytic reactions on supported metal catalyst.

In-situ environmental catalysis was perform ed in an FEI Tecnai F20 field em ission environmental transmission electron microscope operating at $200 \mathrm{kV}$. This $\mathrm{m}$ icroscope is equipped with a Gatan imaging filter and with a dif ferentially pumped environmental cell. A $3 \mathrm{~mm}$ disk of Pt mesh was loaded into a Gatan inc onel heating holder. Ele ctron energy loss spec tra were re corded with the microscope in diffraction m ode with an energy disper sion of $0.1 \mathrm{eV}$. Spectra were acquired from pure gases and gas mixtures for calibration purpose. For the $\mathrm{CO}$ oxidation reaction, $\mathrm{CO}$ and $\mathrm{O}_{2}$ were mixed in a 2:1 $\mathrm{r}$ atio and the catalytic reaction was performed on $\mathrm{Pt} \mathrm{m}$ esh at 1 Torr gas pressure inside the environmental cell. Similar experiments were performed on the same Pt mesh on an ex situ microreactor.

Figure $1 \mathrm{a}$ and $1 \mathrm{~b}$, shows the background subtracted inner-shell spectra from pure $\mathrm{CO}$ and $\mathrm{CO}_{2}$ respectively showing the presence of large $\pi^{*}$ peaks in front of the carbon K-edges $[3,4]$. The $\pi^{*}$ peak positions are calibrated with the $\mathrm{C}$ K-edge from an amorphous carbon film $(284 \mathrm{eV})$. The $\mathrm{C} \pi^{*}$ peaks are at $287.0 \mathrm{eV}$ for $\mathrm{CO}$ and $290.2 \mathrm{eV}$ for $\mathrm{CO}$ 2. Figure $2 \mathrm{a}$, show s the background subtracted energy-loss spectra of $\mathrm{C} \pi^{*}$ peaks obtained while heating the $\mathrm{Pt}$ mesh inside the environmental cell. No catalytic conversion of $\mathrm{CO}$ was observed until $350^{\circ} \mathrm{C}$. A small peak started to appear at $290.2 \mathrm{eV}$ at $400^{\circ} \mathrm{C}$ corresponding to $\mathrm{CO} 2 \pi^{*}$ peak, indicating the form ation of $\mathrm{CO}_{2}$ by the $\mathrm{CO}$ oxidation reaction. This observation confirms that the catalysis has been detected inside the TEM. The $\pi^{*}$ peak at $290.2 \mathrm{eV}$ gradually increases with increasing temperature, which is expected as the increase in temperature increases the catalytic activity. In order to remove the affect of the inconel furnace on catalysis, similar experiments were performed on the holder without the pres ence of Pt $\mathrm{m}$ esh. No $\mathrm{CO}_{2}$ formation was observed even at $500{ }^{\circ} \mathrm{C}$ on the inconel furnace indi cating that the product gas measured is due to the catalytic activity of $\mathrm{Pt}$ for $\mathrm{CO}$ oxidation to $\mathrm{CO}_{2}$. 
Linear curve fitting procedures were used to quantify the gas composition [2]. Figure 1c, shows the normalized reference spectrum from a mixture of $\mathrm{CO}$ and $\mathrm{CO}{ }_{2}$ in $1: 1$ ratio (solid curve). Dotted curve shows the simulated composite spectra from the linear combination of $\mathrm{CO}$ and $\mathrm{CO}_{2}$ individual spectra. The spectra fro $\mathrm{m}$ the gas $\mathrm{m}$ ixture and the composite spectra are alm ost indistinguishable indicating that a good fit has been achieved. Th e ratio of linear coefficients gave a $\mathrm{CO}_{2}$ to $\mathrm{CO}$ ratio of 0.95 which is within $5 \%$ of the expected valu e of 1 . Figure $2 b$, shows the corresponding plot of $\mathrm{CO}_{2}$ to $\mathrm{CO}$ ratio du ring catalysis at differen $\mathrm{t}$ temperatures, obtained from the curve fitting procedures described above. We will present the similar experiments on supported metal catalysts along with the nanoscale structural and chem ical information of the catalyst during the catalytic reaction.

\section{References}

[1] S. Chenna et al., ChemCatChem, n/a, doi: 10.1002/cctc. 201000238.

[2] P.A. Crozier et al., Ultramicroscopy, 111 (2011) 177.

[3] A.P. Hitchcock et al., J. Elec. Spec. Rel. Phenom. 18 (1980) 1.

[4] A.P. Hitchcock et al., J. Elec. Spec. Rel. Phenom. 112 (2000) 9.

[5] The support from the National Science Foundation (NSF-CBET-0553445) and the use of TEM at the John M. Cowley Center for High Resolution Microscopy at Arizona State University are gratefully acknowledged.

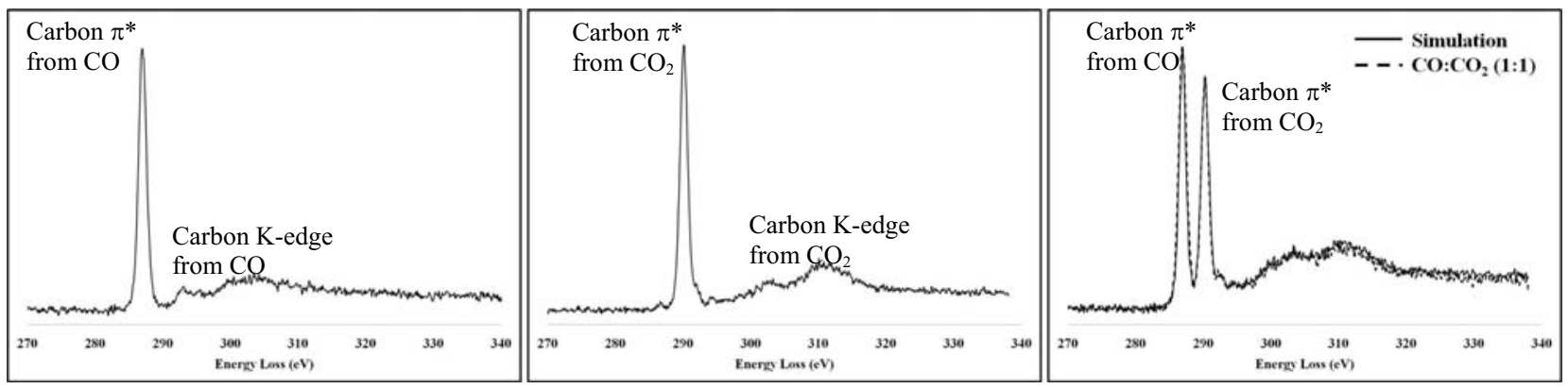

FIG. 1. Background subtracted energy-loss spec tra from a) 1 Torr of CO and b) 1 Torr of $\mathrm{CO}_{2}$ and c) normalized EELS spectra from a mixture of $\mathrm{CO}$ and $\mathrm{CO}{ }_{2}$ in 1:1 ratio at 1 To rr pressure (solid curve). The dotted curve is the linear combination of the individual spectra from both $\mathrm{CO}$ and $\mathrm{CO}_{2}$.
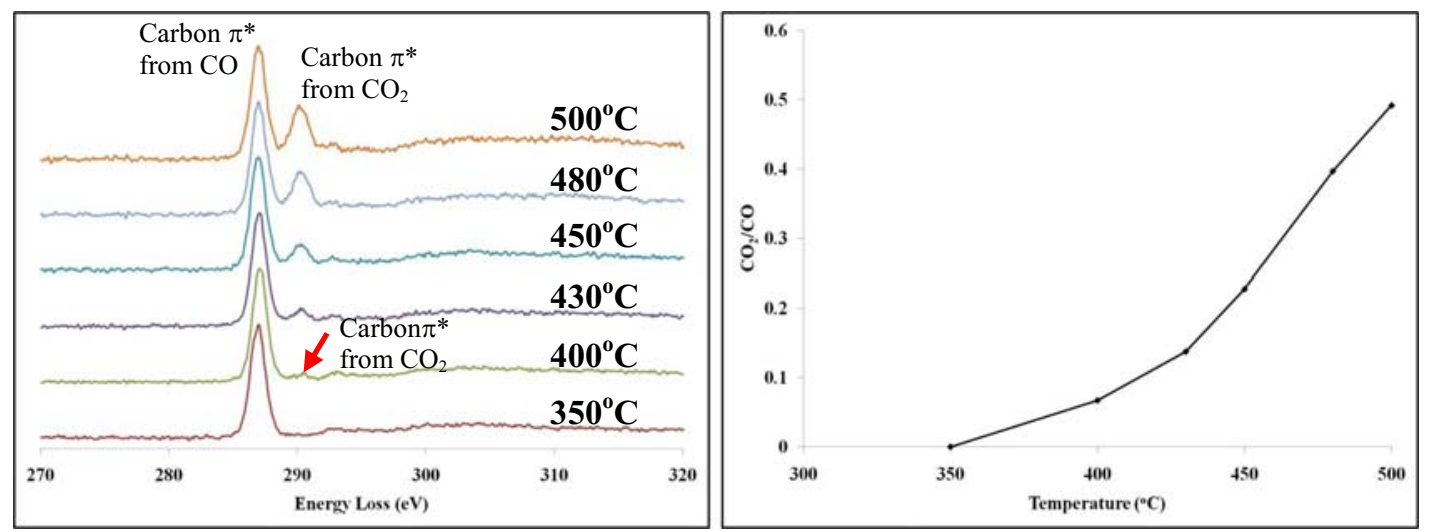

FIG. 2. a) Background subtracted energy-loss spectra acquired at di fferent temperatures during $\mathrm{CO}$ oxidation on $\mathrm{Pt}$ mesh, b) plot showing the ratio of $\mathrm{CO}_{2}$ to $\mathrm{CO}$ with increase in temperature. 